\title{
A!
}

This is an electronic reprint of the original article.

This reprint may differ from the original in pagination and typographic detail.

Sundaria, Ravi; Lehikoinen, Antti; Hannukainen, Antti; Arkkio, Antero

\section{Higher-order finite element modeling of material degradation due to cutting}

Published in:

2017 IEEE International Electric Machines and Drives Conference, IEMDC 2017

DOI:

10.1109/IEMDC.2017.8002278

Published: 03/08/2017

Document Version

Peer reviewed version

Please cite the original version:

Sundaria, R., Lehikoinen, A., Hannukainen, A., \& Arkkio, A. (2017). Higher-order finite element modeling of material degradation due to cutting. In 2017 IEEE International Electric Machines and Drives Conference, IEMDC 2017 [8002278] IEEE. https://doi.org/10.1109/IEMDC.2017.8002278

This material is protected by copyright and other intellectual property rights, and duplication or sale of all or part of any of the repository collections is not permitted, except that material may be duplicated by you for your research use or educational purposes in electronic or print form. You must obtain permission for any other use. Electronic or print copies may not be offered, whether for sale or otherwise to anyone who is not an authorised user. 


\section{Higher-Order Finite Element Modeling of Material Degradation Due to Cutting}

\author{
Ravi Sundaria, Antti Lehikoinen, Antero Arkkio \\ Department of Electrical Engineering and Automation \\ Aalto University \\ Espoo, Finland \\ ravi.sundaria@aalto.fi
}

\author{
Antti Hannukainen \\ Department of Mathematics and System Analysis \\ Aalto Univeristy \\ Espoo, Finland \\ antti.hannukainen@aalto.fi
}

\begin{abstract}
Numerical modeling of energy efficient electrical machines requires accurate and fast calculation of losses. One such loss component is core losses related to magnetic material degradation due to cutting of electrical sheets. This paper analyzes the application of higher order finite elements for precise and computationally efficient modeling of these cutting related losses.
\end{abstract}

\section{INTRODUCTION}

Electrical sheets are cut to their final shape using various techniques (punching, laser cutting), as part of the manufacturing process of electrical machines. These cutting techniques deteriorate the magnetic properties of the material as presented in [1]-[4]. This deterioration further increases the core losses of electrical machines. These losses should be modeled efficiently for the accurate prediction of the electromagnetic losses. A more precise knowledge of these losses will enable designers to design more efficient electrical machines in the future.

Material degradation due to metal cutting in form of grain misorientation was observed by electron backscatter diffraction (EBSD) [2]. Further, micro hardness measurement also confirmed the material degradation as shown in [3]. Moreover, the effect of material degradation on increased iron loss densities was quantified by experiments in [1], [5]. Over the years, many researchers have attempted to model this deterioration in the finite element analysis (FEA) of electrical machines by specifying different layers of degraded materials (degradation profile) in the vicinity of the cut edge as presented in [6], [7]. To provide a more accurate analysis of this magnetic degradation effect [8] tried to use a continuous material model. When this cutting deterioration was modeled in the FEA simulation, iron losses were increased up to 20-30\% [7], [9], [10].

In recent years, machine designers and material engineers have been paying increasing attention on the losses due to cutting and trying to include the cutting effect in the numerical simulation of electrical machines. However, the high cost of computation is one potential barrier in this direction. These

This research work has received funding from the European Research Council under the European Unions Seventh Framework Programme (FP7/20072013) / ERC Grant Agreement n. 339380. cutting related losses are highly sensitive to the distance from the cut edge (range in a few millimeters) and approximated by parabolic or exponential function [5], [6]. Therefore, a very fine mesh is required in the vicinity of the cut edge, which subsequently results in high computational burden in FEA simulations. Clearly, a computationally lighter approach is needed to encourage the inclusion of cutting related losses in electromagnetic computation. Further, it will facilitate the consideration of cutting related losses in the FEA based structural optimization of electrical machines.

To achieve the goal of a computationally efficient finite element approach for inclusion of the cutting effect on magnetic materials, this paper analyzes the use of higher order elements. Higher order elements generally provide better approximation properties whenever the potential function is smooth. Hence, they appear to be an ideal candidate for modeling the edge effects. Moreover, larger elements can be used, in the complex machine geometry areas such as teeth which will result in lower number of unknowns in FEA. First, an analytical expression is derived to account for the material degradation due to the cutting of sheets. It is then further utilized in applying modified material properties at each integration point in a higher order finite element mesh. The accuracy of the obtained results is analyzed by comparing the third order results to densely meshed first-order simulation. Time-stepping analysis of an induction machine is used as an example. Further, a full machine time-harmonic analysis is also performed to analyze the effect of cutting on machine performance and core losses.

\section{METHOD}

This section is divided into two parts. The first part will deal with the derivation of magnetic material degradation profile dependent on the distance from the cut edge and magnetic field intensity. On the other hand, the second part will describe the details of FEA based modeling of the degradation with higher order elements.

1) Derivation of Degradation Profile: The degradation due to the cutting of lamination sheets primarily depends on two parameters: distance from the cut edge $x$ and magnetic field excitation $H$ (effect of excitation frequency is ignored). This paper will use the measurement results from paper [1], which contains $\mathrm{BH}$ characteristics and specific core loss data of 
lamination samples of different widths $(5 \mathrm{~mm}$ to $30 \mathrm{~mm}$ ). Magnetization characteristic of lamination width of $30 \mathrm{~mm}$ is considered as nondegraded. Measurement results are shown in Fig. 1.

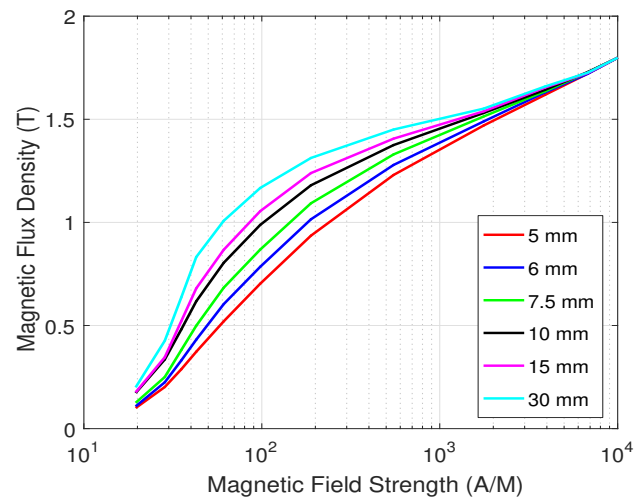

(a)

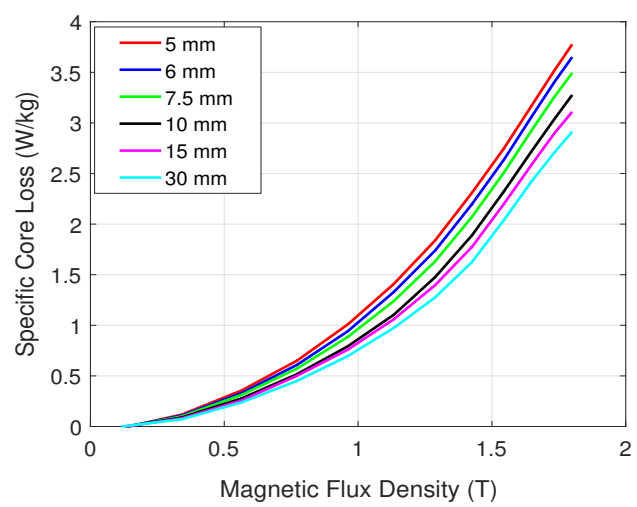

(b)

Fig. 1. (a) Measured magnetization curves (b) Measure specific core loss curves.

Based on [6], [7] the degradation in permeability $\mu(H, x)$ can be represented by exponential or parabolic functions of cut edge distance. This paper model both the magnetic field excitation and cut edge dependency with exponential functions as presented in (1). $\mu_{\mathrm{nd}}$ is permeability of nondegraded material; $a$ and $b$ are fitting parameters.

$$
\mu(H, x)=\mu_{\mathrm{nd}}(H)\left(1-e^{-a x} e^{-b H}\right)
$$

Selection of the exponential function for magnetic field intensity to model the degradation profile of cutting effect is justified in Fig. 2. The Fig. 2 represents that the material degradation decreases as magnetic field intensity increases. Admittedly, the degradation effect has often been observed to disappear or weaken at field strengths below $50 \mathrm{~A} / \mathrm{m}$. As this phenomenon probably has a minor effect in practice, it is ignored in (1).

As each measured $B H$ characteristic represents the average magnetic characteristic (measured permeability $=\mu_{\mathrm{ms}}$ ) of the

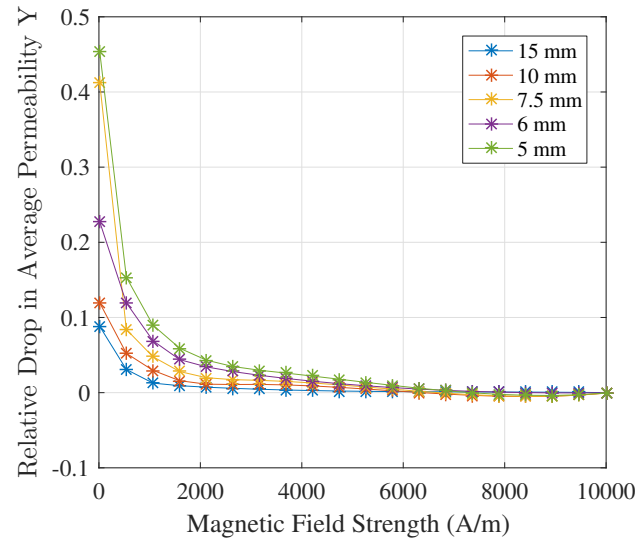

Fig. 2. Relative drop in average permeability of lamination samples of widths: $15 \mathrm{~mm}, 10 \mathrm{~mm}, 7.5 \mathrm{~mm}, 6 \mathrm{~mm}, 5 \mathrm{~mm}$.

sample under measurement; permeability expression in (1) needs to be averaged over the width of the sample $w$.

$$
\begin{aligned}
\mu_{\mathrm{ms}}=\frac{1}{w / 2} & \int_{0}^{w / 2} \mu(H, x) d x \\
& =\frac{1}{w / 2} \int_{0}^{w / 2} \mu_{\mathrm{nd}}(H)\left(1-e^{-a x} e^{-b H}\right) d x
\end{aligned}
$$

Further, simplification of above equation will lead to the expression of relative drop in permeability $Y(H, w)$ as presented in (3).

$$
Y(H, w)=-\left(\frac{\mu_{\mathrm{ms}}(H, w)}{\mu_{\mathrm{nd}}(H)}-1\right)=-\frac{e^{-b H}\left(e^{-a w / 2}-1\right)}{a(w / 2)}
$$

As the measurement was carried out at only $50 \mathrm{~Hz}$ excitation frequency, it is not possible to obtain the core loss components in form of hysteresis, eddy current, and excess losses. Therefore, this paper will proceed with deriving cut distance dependent cumulative local core loss curves based on the measurements. However, it is broadly believed the cutting affects mainly the hysteresis loss components and to some extent excess losses; eddy current losses are largely unaffected [7].

From the Fig. 1b, it is quite clear that core loss density increases near the cut edge. Further, the quadratic polynomial $c B^{2}$ found to fit well with measured core loss curve. These observations lead to the local core loss profile presented in (4); $c$ and $d$ are fitting parameters.

$$
p(B(H, x), x)=c\left(1+e^{-d x}\right) B^{2}(H, x)
$$

Here, the localized magnetic flux density is considered as $B(H, x)=\mu(H, x) H$. Further, this localized core loss density (4) needs to be integrated over the width of lamination to obtained the measured core loss density $p_{\mathrm{ms}}$.

$$
p_{\mathrm{ms}}=\frac{1}{w / 2} \int_{0}^{w / 2} p(B(H, x), x) d x
$$




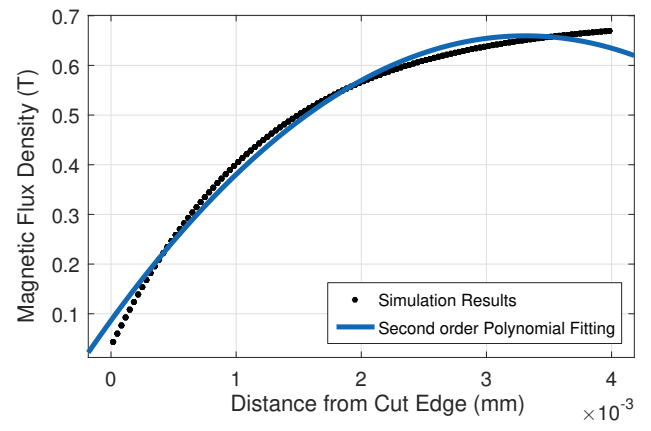

Fig. 3. Second order polynomial fitting with the variation of magnetic flux density in a half stator tooth as a function of cut-edge distance.

2) Finite Element Formulation with Higher Order Element: The appropriate order of higher order finite element needs to be selected to model the cutting effect in FEA. The order of a finite element depends on the variation of magnetic flux density inside one element. Therefore, the permeability expression (1) is applied to the nonlinear FEA simulation of very dense first order mesh with continuous material model [8]. From the results, the behavior of the flux density is evaluated as a function of the distance from the cut edge.

Based on the nonlinear finite element simulation, second order polynomial fits reasonably to flux density profile as shown in the Fig. 3. Therefore, third order elements seem to be a natural choice for modeling the presented formulation for the cutting effects. Further, stator tooth region is selected to analyze the accuracy of higher order simulation, since it can be assumed to be one of the most affected areas due to the cutting of electrical sheets. Therefore, we arrived at the conclusion that third order elements covering a tooth width with adequate number of integration points should perform the numerical simulation accurately. For electromagnetic finite element analysis of tooth, well known $A V$ formulation based on magnetic vector potential is used.

$$
\mathbf{S}(\mathbf{a}, x) \mathbf{a}=\mathbf{f}
$$

Here, the stiffness matrix $\mathbf{S}$ is dependent on magnetic vector potential a and cut-distance $x$. $\mathbf{f}$ is the source vector representing the effect of supply current density. Non-linearity in (6) is handled by Newton Raphson method.

Third order elements achieve good accuracy as later presented in the result section. Therefore, this paper proceed with application of the higer order finite element formulation together with the circuit equations for a time harmonic case to study complete machine geometry. Studied time harmonic model of cage induction machine is based on [11]. The contribution of stator end winding impedance was taken into account with the help of circuit equations.

$$
\left[\begin{array}{cc}
\mathbf{S}+\tilde{\mathbf{M}} & \left(\mathbf{D}^{s}\right)^{T} \mathbf{K}^{T} \\
\mathbf{K D}^{s} & \tilde{\mathbf{G}}^{s}
\end{array}\right] \cdot\left[\begin{array}{c}
\tilde{\mathbf{a}} \\
\tilde{\mathbf{i}}^{s}
\end{array}\right]=\left[\begin{array}{c}
0 \\
-\tilde{\mathbf{V}}^{s}
\end{array}\right]
$$

Nonlinear system of equations in (7) are solved for unknowns: magnetic vector potential $\tilde{\mathbf{a}}$ and supply current $\tilde{\mathbf{i}}^{s}$.
Here symbol ${ }^{\sim}$ denote the complex nature of matrices. Further, $\mathbf{S}$ and $\tilde{\mathbf{M}}$ are Stiffness and Mass matrices. The stator flux linkage is represented by the matrix $\mathbf{D}^{s}$. Moreover, the stator winding impedance is included in form of matrix $\tilde{\mathbf{G}}^{s}$ with voltage source $\tilde{\mathbf{V}}^{s}$.

\section{RESULTS}

1) Fitting results: Parameters for the modified permeability expression and core loss were fitted with the help of nonlinear least-squares solver of MATLAB. Based on the curve fitting results, values of parameters are: $a=795(1 / \mathrm{m}), b=0.001664$ $(\mathrm{A} / \mathrm{m})^{-1}, c=0.835$ and $d=925$. Fitted magnetization and specific loss curves of lamination samples are shown in Fig. 4. Further, the fitting is checked to judge mean error in magnetic

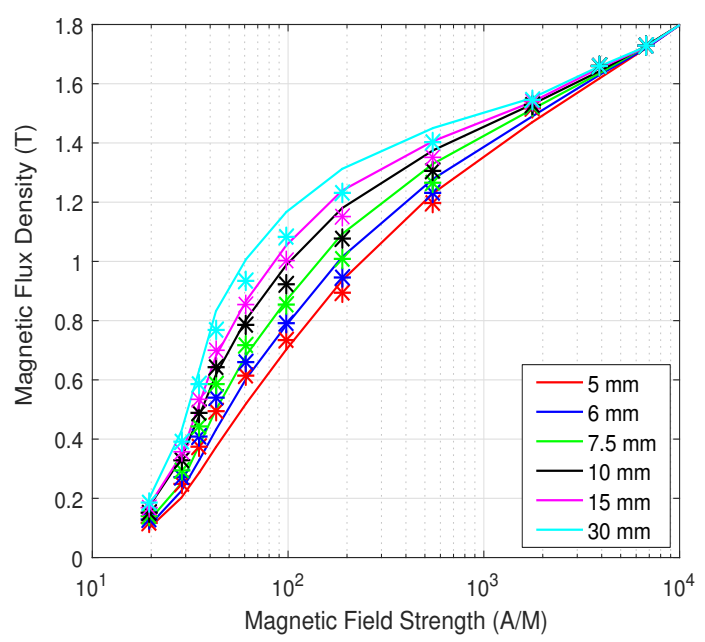

(a)

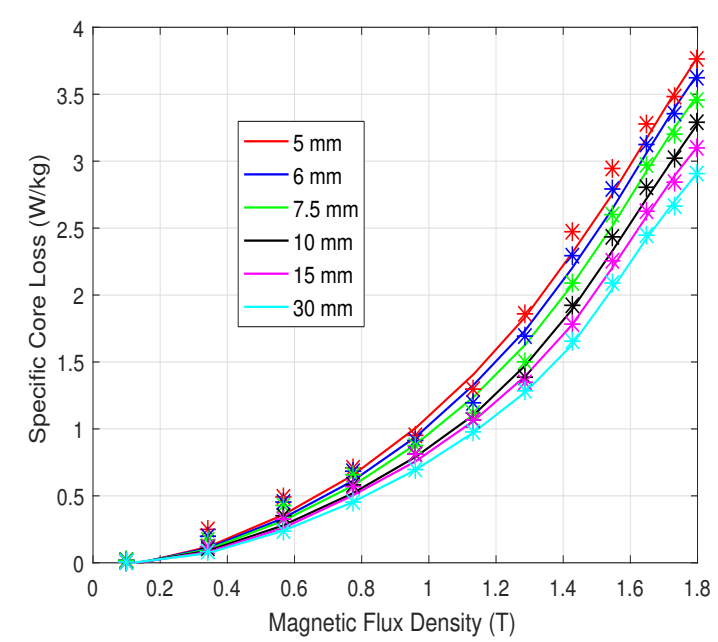

(b)

Fig. 4. (a) Magnetic flux density as a function of magnetic field strength. (b) Specific core loss as a function of magnetic flux density. The experimental curves together with the optimal fit given by markers. As per [1] lamination samples of width $5 \mathrm{~mm}$ to $30 \mathrm{~mm}$ were considered.

flux density $D_{\mathrm{B}}$ and core loss density $D_{\mathrm{p}}$.

$$
D_{\mathrm{B}}=\frac{1}{N_{m} N_{n}} \sqrt{\sum_{m=1}^{N_{m}} \sum_{n=1}^{N_{n}}\left(\Delta B_{m n}\right)^{2}}
$$




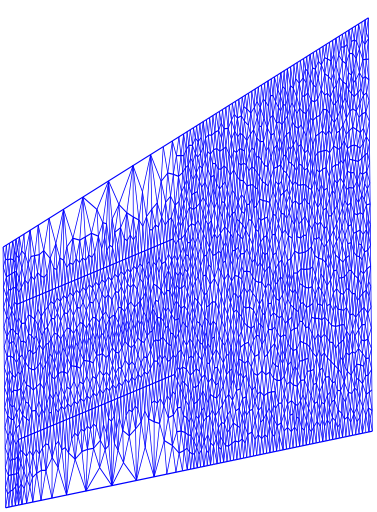

(a)

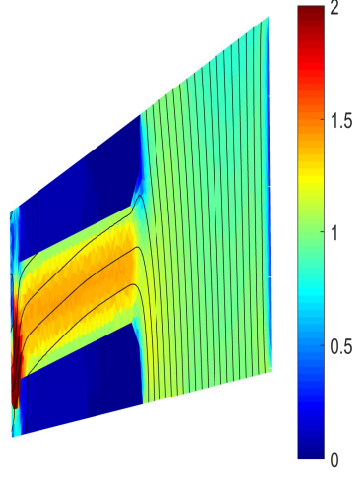

(b)

Fig. 5. (a) Highly dense first order mesh (b) Magnetic flux density at a timestep.

$$
D_{\mathrm{p}}=\frac{1}{N_{m} N_{n}} \sqrt{\sum_{m=1}^{N_{m}} \sum_{n=1}^{N_{n}}\left(\Delta p_{m n}\right)^{2}}
$$

Logarithmic scaled 12 data points of magnetic field intensity $\left(N_{n}=12\right)$ between $10 \mathrm{~A} / \mathrm{m}$ to $10000 \mathrm{~A} / \mathrm{m}$ along with six sample lamination widths $\left(N_{m}=6 ; 5 \mathrm{~mm}-30 \mathrm{~mm}\right)$ are chosen which resulted in mean errors $D_{\mathrm{B}}=0.0045 \mathrm{~T}$ and $D_{\mathrm{p}}=0.0078 \mathrm{~W} / \mathrm{kg}$.

2) Accuracy of higher order finite element modeling: Time stepping finite element analysis of a three phase cage induction machine at full load is performed. A tooth of the studied machine was extracted to analyze the cutting effect. As a very dense mesh is required for the accurate calculation of cutting effects, a highly dense first order mesh was considered as a reference case for this study (Fig. 5a). Further two third order meshes, dense and coarse (Fig. 6a and 7a) were chosen for comparison purposes.

Dirichlet boundary conditions were used. The boundary data was obtained by simulating the whole cross-section without considering the punching effects. Two periods were analyzed, with 200 steps per period. Error in vector potential solution

TABLE I

MESH DATA

\begin{tabular}{|l|l|l|}
\hline Mesh & Elements & Nodes \\
\hline First Order & 4328 & 2256 \\
\hline $\begin{array}{l}\text { Third Order } \\
\text { Dense }\end{array}$ & 503 & 2350 \\
\hline $\begin{array}{l}\text { Third Order } \\
\text { Coarse }\end{array}$ & 68 & 343 \\
\hline
\end{tabular}

of FEA is then calculated to judge the accuracy of high order elements. Let, $A_{e l, t}^{1}$ be the vector potential solution of first order mesh at time step $t$ at the centroid of first order triangular element $e l$ of area $\Omega_{e l}$; at the same position the corresponding solutions in third order dense and coarse meshes are $A_{e l, t}^{3 d}$, $A_{e l, t}^{3 c}$ respectively.

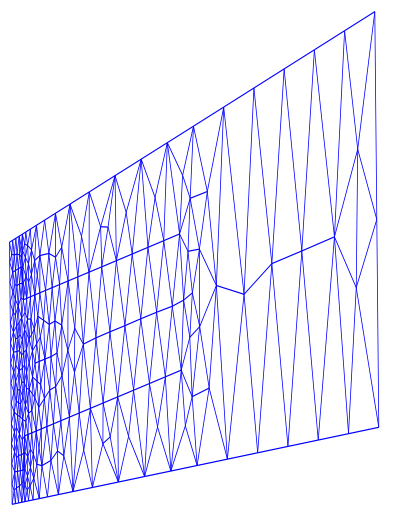

(a)

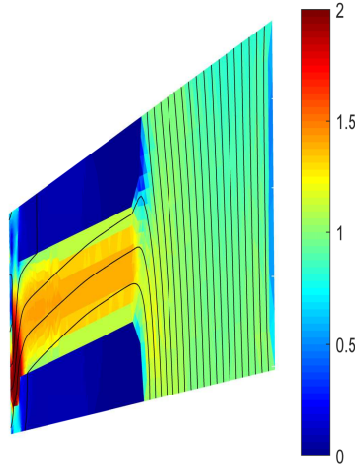

(b)
Fig. 6. (a) Third order dense mesh (b) Magnetic flux density at a timestep.

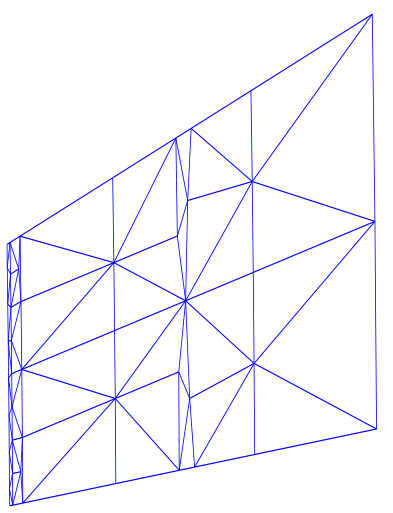

(a)

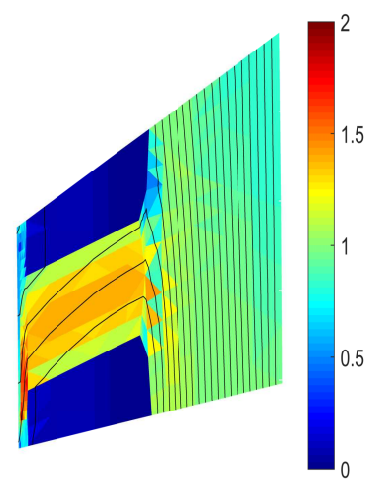

(b)
Fig. 7. (a) Third order coarse mesh (b) Magnetic flux density at a timestep.

First, the error at a timestep $t$ is calculated as $E_{t}(10)$ and then average error across all timesteps are obtained as $G$ (11).

$$
\begin{gathered}
E_{t}=\frac{\sqrt{\sum_{e l=1}^{N}\left[\left(A_{e l, t}^{3 d}-A_{e l, t}^{1}\right) \Omega_{e l}\right]^{2}}}{\sqrt{\sum_{e l=1}^{N}\left(A_{e l, t}^{1} \Omega_{e l}\right)^{2}}} \\
G=\frac{\sqrt{\sum_{t=1}^{400} E_{t}^{2}}}{400}
\end{gathered}
$$

As a result of time-stepping nonlinear FEA, calculated $G$ for dense third order mesh (Fig. 6a) and coarse third order mesh (Fig. 7a) are calculated as $1.7853 \cdot 10^{-05}$ and $2.84 \cdot 10^{-04}$ respectively.

3) Time harmonic simulation of induction machine: As the comparative simulation results of third order coarse mesh (Fig. 7a) were found satisfactory, a similar coarse mesh was used to evaluate a complete machine geometry. To analyze the effect of cutting on the core losses and force computation a time harmonic model of $37 \mathrm{~kW}$ induction machine (Table II) was studied. The mesh consists of 1516 third order elements and 6901 nodes. At the rated load the core losses in stator core are computed with the help of derived local specific core 
TABLE II

MOTOR DATA

\begin{tabular}{|l|l|}
\hline Shaft Power & $37 \mathrm{~kW}$ \\
\hline Voltage & $400 \mathrm{~V}$ \\
\hline Frequency & $50 \mathrm{~Hz}$ \\
\hline Connection & Star \\
\hline Pole pairs & 2 \\
\hline Stator outer diameter & $310 \mathrm{~mm}$ \\
\hline Stator inner diameter & $200 \mathrm{~mm}$ \\
\hline Air gap & $0.8 \mathrm{~mm}$ \\
\hline Number of stator slots & 48 \\
\hline Number of rotor slots & 40 \\
\hline
\end{tabular}

loss curves. Cutting of electrical sheets increases the specific core loss density near the cut-edge and therefore result in an overall increase in core losses. The increase in stator core losses for the studied motor was about $11 \%$.

Moreover, the modeled decrease in permeability will force the magnetic flux away from the cut-edge. This effect in the vicinity of cut-edge is clearly observed near rotor bar edges as presented in Fig. 8c in form of difference in magnetic flux density distribution. No major effect on the output torque and load current were observed due to cutting. Similar cutting related continuous material model was applied to a permanent magnet machine, researchers have not observed a significant difference in the machine torque [9]. Furthermore, similar cage induction machine with a degraded material layer along the cut-edge was studied in [10]. Effect of the cutting on the output torque was relatively small. Core losses corresponding to fundamental excitation frequency from the rotor side are considered to be insignificant for comparison purpose in the studied time-harmonic case. However, in the practical case, we will need localized core loss components to account the losses corresponding to slot harmonics.

TABLE III

TIME HARMONIC SIMULATION AT FULL LOAD

\begin{tabular}{|l|l|l|l|}
\hline Parameter & Nondegraded & Degraded & \%Difference \\
\hline $\begin{array}{l}\text { Stator Cur- } \\
\text { rent (A) }\end{array}$ & 73.14 & 73.44 & 0.41 \\
\hline $\begin{array}{l}\text { Torque } \\
\text { (Nm) }\end{array}$ & 242.02 & 241.28 & -0.30 \\
\hline $\begin{array}{l}\text { Stator Core } \\
\text { Loss (W) }\end{array}$ & 136.34 & 151.95 & 11.45 \\
\hline Slip (\%) & 1.35 & 1.35 & \\
\hline
\end{tabular}

\section{CONCLUSION}

This paper applied higher order elements in the modeling of losses related to the cutting of electrical sheets. First, analytical expressions to account for the effect of cutting on magnetization and core loss density were derived. Further, third order elements appeared to be a suitable candidate for higher order FEA to model cutting related losses. The

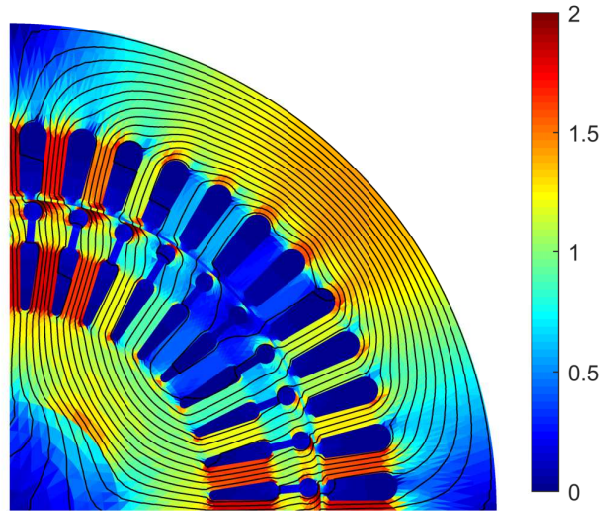

(a)

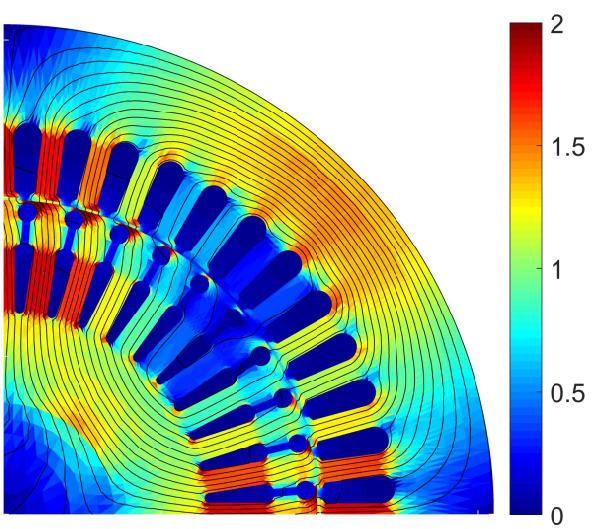

(b)

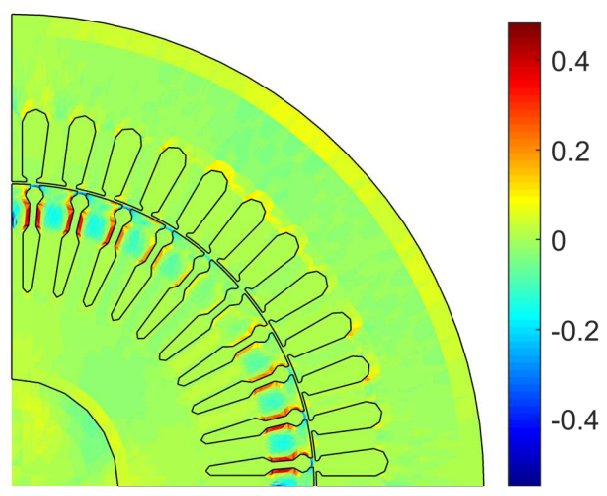

(c)

Fig. 8. Magnetic Flux density distribution (a) Without cutting effect (b) With cutting effect (c) Respective difference.

simulation results with higher order elements were compared with highly dense first order mesh to examine the accuracy of the solution. Satisfactory results were observed in timestepping finite element based analysis of an extracted tooth of an induction machine at full load.

Moreover, time-harmonic simulation of a full induction machine with presented higher order formulation was also studied. Significant effect on the core losses has been observed. 
Further, it is worth to note that the measurement results analyzed in this paper are related to the punching of electrical sheets and laser cutting proved to have even more effect on magnetization and core loss characteristics [12]. Even with punching, as punching tool degrades the effect of cutting increases. As the machine designers are moving towards very high-efficiency machines for future needs, the inclusion of cutting related losses will be important at the design stage. Clearly, use of higher order elements will remove the need to have a very fine or a multi-layered mesh near the cut-edge and therefore enable the FEA simulations more suitable for inclusion of the cutting related losses.

\section{REFERENCES}

[1] A. Schoppa, J. Schneider, and C.-D. Wuppermann, "Influence of the manufacturing process on the magnetic properties of non-oriented electrical steels," Journal of Magnetism and Magnetic Materials, vol. 215, pp. 74-78, 2000.

[2] M. Bali and A. Muetze, "Modeling the effect of cutting on the magnetic properties of electrical steel sheets," IEEE Transactions on Industrial Electronics, vol. 64, no. 3, pp. 2547-2556, March 2017.

[3] F. Ossart, E. Hug, O. Hubert, C. Buvat, and R. Billardon, "Effect of punching on electrical steels: Experimental and numerical coupled analysis," IEEE Trans. Magn., vol. 36, no. 5, pp. 3137-3140, 2000.

[4] T. Nakata, M. Nakano, and K. Kawahara, "Effects of stress due to cutting on magnetic characteristics of silicon steel," IEEE translation journal on magnetics in Japan, vol. 7, no. 6, pp. 453-457, 1992.

[5] L. Vandenbossche, S. Jacobs, F. Henrotte, and K. Hameyer, "Impact of cut edges on magnetization curves and iron losses in e-machines for automotive traction," in The 25th World Battery, Hybrid and Fuel Cell Electric Vehicle Symposium \& Exhibition, 2010.

[6] M. Bali, H. De Gersem, and A. Muetze, "Finite-element modeling of magnetic material degradation due to punching," IEEE Trans. Magn., vol. 50, no. 2, pp. 745-748, 2014.

[7] L. Vandenbossche, S. Jacobs, X. Jannot, M. McClelland, J. Saint-Michel, and E. Attrazic, "Iron loss modelling which includes the impact of punching, applied to high-efficiency induction machines," in 2013 3rd International Electric Drives Production Conference (EDPC), Oct 2013 , pp. $1-10$.

[8] S. Elfgen, S. Steentjes, S. Böhmer, D. Franck, and K. Hameyer, "Continuous local material model for cut edge effects in soft magnetic materials," IEEE Trans. Magn., vol. 52, no. 5, pp. 1-4, 2016.

[9] S. Elfgen, S. Steentjes, S. Bhmer, D. Franck, and K. Hameyer, "Influences of material degradation due to laser cutting on the operating behaviour of pmsm using a continuous local material model," in 2016 XXII International Conference on Electrical Machines (ICEM), Sept 2016, pp. $1835-1840$

[10] P. Rasilo, U. Aydin, T. P. Holopainen, and A. Arkkio, "Analysis of iron losses on the cutting edges of induction motor core laminations," in 2016 XXII International Conference on Electrical Machines (ICEM), Sept 2016, pp. 1312-1317.

[11] A. Arkkio, "Analysis of induction motors based on the numerical solution of the magnetic field and circuit equations," http://lib.tkk.fi/Diss/ 198X/isbn951226076X/, 1987, [PhD Thesis].

[12] K. Bourchas, A. Stening, J. Soulard, A. Broddefalk, M. Lindenmo, M. Dahln, and F. Gyllensten, "Influence of cutting and welding on magnetic properties of electrical steels," in 2016 XXII International Conference on Electrical Machines (ICEM), Sept 2016, pp. 1815-1821. 